\title{
Antiperiodic Solutions for Liénard-Type Differential Equation with $p$-Laplacian Operator
}

\author{
Taiyong Chen, Wenbin Liu, and Cheng Yang \\ Department of Mathematics, China University of Mining and Technology, Xuzhou 221008, China \\ Correspondence should be addressed to Taiyong Chen, taiyongchen@163.com
}

Received 2 March 2010; Revised 22 July 2010; Accepted 19 August 2010

Academic Editor: Irena Rachůnkova

Copyright (C) 2010 Taiyong Chen et al. This is an open access article distributed under the Creative Commons Attribution License, which permits unrestricted use, distribution, and reproduction in any medium, provided the original work is properly cited.

The existence of antiperiodic solutions for Liénard-type and Duffing-type differential equations with $p$-Laplacian operator has been studied by using degree theory. The results obtained improve and enrich some known works to some extent.

\section{Introduction}

Antiperiodic problems arise naturally from the mathematical models of various of physical processes (see [1, 2]), and also appear in the study of partial differential equations and abstract differential equations (see [3-5]). For instance, electron beam focusing system in travelling-wave tube's theories is an antiperiodic problem (see [6]).

During the past twenty years, antiperiodic problems have been studied extensively by numerous scholars. For example, for first-order ordinary differential equations, a Massera's type criterion was presented in [7] and the validity of the monotone iterative technique was shown in [8]. Moreover, for higher-order ordinary differential equations, the existence of antiperiodic solutions was considered in [9-12]. Recently, existence results were extended to antiperiodic boundary value problems for impulsive differential equations (see [13]), and antiperiodic wavelets were discussed in [14].

Wang and $\mathrm{Li}$ (see [15]) discussed the existence of solutions of the following antiperiodic boundary value problem for second-order conservative system:

$$
q^{\prime \prime}=u(t, q), \quad q(0)=-q(T), \quad q^{\prime}(0)=-q^{\prime}(T)
$$

using of the main assumption as follows: 
$\left(A_{1}\right)$ There exist constants $0 \leq c<8$ and $M>0$, such that

$$
|u(t, q)| \leq \frac{c}{T^{2}}|q|+M, \quad \forall t \in[0, T], q \in \mathbb{R}
$$

The turbulent flow in a porous medium is a fundamental mechanics problem. For studying this type of problems, Leibenson (see [16]) introduced the following $p$-Laplacian equation:

$$
\left(\phi_{p}\left(x^{\prime}\right)\right)^{\prime}=f\left(t, x, x^{\prime}\right),
$$

where $\phi_{p}(s)=|s|^{p-2} s, p>1$. Obviously, the inverse operator of $\phi_{p}$ is $\phi_{q}$, where $q>1$ is a constant such that $1 / p+1 / q=1$.

Notice that, when $p=2$, the nonlinear operator $\left(\phi_{p}\left(x^{\prime}\right)\right)^{\prime}$ reduces to the linear operator $x^{\prime \prime}$.

In the past few decades, many important results relative to (1.3) with certain boundary conditions have been obtained. We refer the reader to [17-20] and the references cited therein. However, to the best of our knowledge, there exist relatively few results for the existence of antiperiodic solutions of (1.3). Moreover, it is well known that the existence of antiperiodic solutions plays a key role in characterizing the behavior of nonlinear differential equations (see [21]). Thus, it is worthwhile to continue to investigate the existence of antiperiodic solutions for (1.3).

A primary purpose of this paper is to study the existence of antiperiodic solutions for the following Liénard-type $p$-Laplacian equation:

$$
\left(\phi_{p}\left(x^{\prime}\right)\right)^{\prime}+f(x) x^{\prime}+g(t, x)=e(t)
$$

and antiperiodic solutions with symmetry for Duffing-type $p$-Laplacian equation as follows:

$$
\left(\phi_{p}\left(x^{\prime}\right)\right)^{\prime}+g(t, x)=e(t)
$$

where $f, e \in C(\mathbb{R}, \mathbb{R}), g \in C\left(\mathbb{R}^{2}, \mathbb{R}\right)$ with $f(-x) \equiv f(x), g(t+\pi,-x) \equiv-g(t, x)$, and $e(t+\pi) \equiv$ $-e(t)$. That is, we will prove that (1.4) or (1.5) has at least one solution $x(t)$ satisfying

$$
x(t+\pi)=-x(t), \quad \forall t \in \mathbb{R} .
$$

Note that, $x(t)$ is also a $2 \pi$-periodic solution of (1.4) or (1.5) if $x(t)$ is a $\pi$-antiperiodic solution of (1.4) or (1.5). Hence, from the arguments in this paper, we can also obtain the existence results of periodic solutions for above equations.

The rest of this paper is organized as follows. Section 2 contains some necessary preliminaries. In Section 3, we establish some sufficient conditions for the existence of antiperiodic solutions of (1.4), basing on Leray-Schauder principle. Then, in Section 4, we obtain two existence results of antiperiodic solutions with symmetry for (1.5). Finally, in Section 5, some explicit examples are given to illustrate the main results. Our results are different from those of bibliographies listed above. 


\section{Preliminaries}

For convenience, we introduce some notations as follows:

$$
\begin{aligned}
C^{k, 2 \pi} & =\left\{x \in C^{k}(\mathbb{R}, \mathbb{R}): x(t+2 \pi) \equiv x(t)\right\}, \\
C^{k, \pi} & =\left\{x \in C^{k, 2 \pi}: x(t+\pi) \equiv-x(t)\right\}, \\
C_{0}^{k, \pi} & =\left\{x \in C^{k, \pi}: x(-t) \equiv x(t)\right\}, \\
C_{1}^{k, \pi} & =\left\{x \in C^{k, \pi}: x(-t) \equiv-x(t)\right\} \\
\|x\|_{\infty} & =\max _{t \in[0,2 \pi]}|x(t)|, \quad x \in C^{0,2 \pi}, \\
\|x\|_{C^{k}} & =\max _{i \in\{0,1, \ldots, k\}}\left\{\left\|x^{(i)}\right\|_{\infty}\right\}, \quad x \in C^{k, 2 \pi},
\end{aligned}
$$

and $\|\cdot\|_{p}$ denotes norm in $L^{p}([0,2 \pi], \mathbb{R})$.

For each $x \in C^{0, \pi}$, there exists the following Fourier series expansion:

$$
x(t)=\sum_{i=0}^{\infty}\left[a_{2 i+1} \cos (2 i+1) t+b_{2 i+1} \sin (2 i+1) t\right]
$$

where $a_{2 i+1}, b_{2 i+1} \in \mathbb{R}$. Let us define the mapping $J: C^{0, \pi} \rightarrow C^{1, \pi}$ by

$$
\begin{aligned}
(J x)(t) & =\int_{0}^{t} x(s) d s-\sum_{i=0}^{\infty} \frac{b_{2 i+1}}{2 i+1} \\
& =\sum_{i=0}^{\infty}\left[\frac{a_{2 i+1}}{2 i+1} \sin (2 i+1) t-\frac{b_{2 i+1}}{2 i+1} \cos (2 i+1) t\right], \quad \forall t \in \mathbb{R}
\end{aligned}
$$

Notice that, $x \in C_{0}^{0, \pi}$ may be written as Fourier series as follows:

$$
x(t)=\sum_{i=0}^{\infty} a_{2 i+1} \cos (2 i+1) t
$$

and $x \in C_{1}^{0, \pi}$ may be written as the following Fourier series:

$$
x(t)=\sum_{i=0}^{\infty} b_{2 i+1} \sin (2 i+1) t
$$


We define the mapping $J_{0}: C_{0}^{0, \pi} \rightarrow C_{1}^{1, \pi}$ by

$$
\left(J_{0} x\right)(t)=\int_{0}^{t} x(s) d s=\sum_{i=0}^{\infty} \frac{a_{2 i+1}}{2 i+1} \sin (2 i+1) t, \quad \forall t \in \mathbb{R}
$$

and the mapping $J_{1}: C_{1}^{0, \pi} \rightarrow C_{0}^{1, \pi}$ by

$$
\left(J_{1} x\right)(t)=\int_{0}^{t} x(s) d s-\sum_{i=0}^{\infty} \frac{b_{2 i+1}}{2 i+1}=-\sum_{i=0}^{\infty} \frac{b_{2 i+1}}{2 i+1} \cos (2 i+1) t, \quad \forall t \in \mathbb{R}
$$

It is easy to prove that the mappings $J, J_{0}, J_{1}$ are completely continuous by using ArzelàAscoli theorem.

Next, we introduce a Wirtinger inequality (see [22]) and a continuation theorem (see $[23,24])$ as follows.

Lemma 2.1 (Wirtinger inequality). For each $x \in W^{1, p}([0,2 \pi], \mathbb{R})$ such that $x(0)=x(2 \pi)$ and $\int_{0}^{2 \pi}|x(t)|^{p-2} x(t) d t=0$, one has

$$
\lambda_{1}\|x\|_{p}^{p} \leq\left\|x^{\prime}\right\|_{p^{\prime}}^{p}
$$

where

$$
\lambda_{1}=\left(\frac{\pi_{p}}{\pi}\right)^{p}, \quad \pi_{p}=\frac{2 \pi(p-1)^{1 / p}}{p \sin (\pi / p)} .
$$

Lemma 2.2 (Continuation theorem). Let $\Omega$ be open-bounded in a linear normal space X. Suppose that $f$ is a completely continuous field on $\bar{\Omega}$. Moreover, assume that the Leray-Schauder degree

$$
\operatorname{deg}(f, \Omega, p) \neq 0, \quad \text { for } p \in X \backslash f(\partial \Omega)
$$

Then equation $f(x)=p$ has at least one solution in $\Omega$.

\section{Antiperiodic Solutions for (1.4)}

In this section, an existence result of antiperiodic solutions for (1.4) will be given.

Theorem 3.1. Assume that

$\left(H_{1}\right)$ there exists a nonnegative function $\alpha \in C\left(\mathbb{R}, \mathbb{R}^{+}\right)$such that

$$
\limsup _{|x| \rightarrow+\infty} \frac{|g(t, x)|}{|x|^{p-1}}=\alpha(t), \quad \forall t \in \mathbb{R}
$$


where

$$
\|\alpha\|_{\infty}<\left(\frac{\pi_{p}}{\pi}\right)^{p}\left(=\lambda_{1}\right)
$$

Then (1.4) has at least one antiperiodic solution.

Remark 3.2. When $p=2, \lambda_{1}$ is equal to 1 . It is easy to see that condition $\left(A_{1}\right)$ in [15] is stronger than condition $\left(H_{1}\right)$ of Theorem 3.1.

For making use of Leray-Schauder degree theory to prove the existence of antiperiodic solutions for (1.4), we consider the homotopic equation of (1.4) as follows:

$$
\left(\phi_{p}\left(x^{\prime}\right)\right)^{\prime}=-\lambda f(x) x^{\prime}-\lambda g(t, x)+\lambda e(t), \quad \lambda \in[0,1]
$$

Define the operator $L_{p}: D\left(L_{p}\right) \subset C^{1, \pi} \rightarrow L^{1}([0,2 \pi], \mathbb{R})$ by

$$
\left(L_{p} x\right)(t)=\left(\phi_{p}\left(x^{\prime}(t)\right)\right)^{\prime}, \quad \forall t \in \mathbb{R},
$$

where

$$
D\left(L_{p}\right)=\left\{x \in C^{1, \pi}: \phi_{p}\left(x^{\prime}(t)\right) \text { is absolutely continuous on } \mathbb{R}\right\} .
$$

Let $N: C^{1, \pi} \rightarrow L^{1}([0,2 \pi], \mathbb{R})$ be the Nemytski operator

$$
(N x)(t)=-f(x(t)) x^{\prime}(t)-g(t, x(t))+e(t), \quad \forall t \in \mathbb{R}
$$

Obviously, the operator $L_{p}$ is invertible and the antiperiodic problem of (3.3) is equivalent to the operator equation

$$
L_{p} x=\lambda N x, \quad x \in D\left(L_{p}\right)
$$

We begin with some lemmas below.

Lemma 3.3. Suppose that the assumption $\left(H_{1}\right)$ is true. Then the antiperiodic solution $x(t)$ of (3.3) satisfies

$$
\left\|x^{\prime}\right\|_{p} \leq K_{1}
$$

where $K_{1}$ is a positive constant only dependent of $\lambda_{1}$ and $\|e\|_{\infty}$. 
Proof. Multiplying the both sides of (3.3) with $x(t)$ and integrating it over $[0,2 \pi]$, we get

$$
\begin{aligned}
\int_{0}^{2 \pi}\left(\phi_{p}\left(x^{\prime}(t)\right)\right)^{\prime} x(t) d t= & -\lambda \int_{0}^{2 \pi} f(x(t)) x^{\prime}(t) x(t) d t \\
& -\lambda \int_{0}^{2 \pi} g(t, x(t)) x(t) d t+\lambda \int_{0}^{2 \pi} e(t) x(t) d t .
\end{aligned}
$$

Noting that

$$
\int_{0}^{2 \pi}\left(\phi_{p}\left(x^{\prime}(t)\right)\right)^{\prime} x(t) d t=-\int_{0}^{2 \pi} \phi_{p}\left(x^{\prime}(t)\right) x^{\prime}(t) d t=-\left\|x^{\prime}\right\|_{p}^{p}
$$

and $\int_{0}^{2 \pi} f(x(t)) x(t) x^{\prime}(t) d t=0$, we have

$$
\left\|x^{\prime}\right\|_{p}^{p} \leq \int_{0}^{2 \pi}|g(t, x(t))||x(t)| d t+\int_{0}^{2 \pi}|e(t)||x(t)| d t .
$$

By hypothesis $\left(H_{1}\right)$, there exists a nonnegative constant $\beta$ such that

$$
|g(t, x)| \leq \alpha(t)|x|^{p-1}+\beta, \quad \forall t, x \in \mathbb{R} .
$$

Thus, from (3.11), we have

$$
\left\|x^{\prime}\right\|_{p}^{p} \leq\|\alpha\|_{\infty}\|x\|_{p}^{p}+\left(\beta+\|e\|_{\infty}\right) \int_{0}^{2 \pi}|x(t)| d t .
$$

That is,

$$
\left\|x^{\prime}\right\|_{p}^{p} \leq\|\alpha\|_{\infty}\|x\|_{p}^{p}+K_{2}\|x\|_{p}
$$

where $K_{2}=(2 \pi)^{1 / q}\left(\beta+\|e\|_{\infty}\right)$.

For each $x \in C^{1, \pi}$, we get

$$
\int_{0}^{2 \pi} x(t) d t=\int_{0}^{\pi} x(t) d t+\int_{0}^{\pi} x(t+\pi) d t=0
$$

Similarly, we obtain that

$$
\begin{gathered}
\int_{0}^{2 \pi} x^{\prime}(t) d t=0 \\
\int_{0}^{2 \pi}|x(t)|^{p-2} x(t) d t=0
\end{gathered}
$$


Basing on Lemma 2.1, it can be shown from (3.17) and (3.14) that

$$
\left\|x^{\prime}\right\|_{p}^{p} \leq \frac{\|\alpha\|_{\infty}}{\lambda_{1}}\left\|x^{\prime}\right\|_{p}^{p}+\frac{K_{2}}{\lambda_{1}^{1 / p}}\left\|x^{\prime}\right\|_{p}
$$

Let $K_{1}=\left(K_{2} \lambda_{1}^{1 / q} /\left(\lambda_{1}-\|\alpha\|_{\infty}\right)\right)^{1 /(p-1)}>0$, then

$$
\left\|x^{\prime}\right\|_{p} \leq K_{1}
$$

The proof is complete.

Lemma 3.4. Suppose that the assumption $\left(H_{1}\right)$ is true. Then, for the possible antiperiodic solution $x(t)$ of (3.3), there exists a prior bounds in $C^{1, \pi}$, that is, $x(t)$ satisfies

$$
\|x\|_{C^{1}} \leq T_{1}
$$

where $T_{1}$ is a positive constant independent of $\lambda$.

Proof. By (3.15), there exists $t_{1} \in[0,2 \pi]$ such that $x\left(t_{1}\right)=0$. Hence, (3.8) yields that

$$
\|x\|_{\infty} \leq \int_{0}^{2 \pi}\left|x^{\prime}(t)\right| d t \leq(2 \pi)^{1 / q}\left\|x^{\prime}\right\|_{p} \leq(2 \pi)^{1 / q} K_{1}:=K_{3} .
$$

Letting

$$
\begin{gathered}
K_{4}=\max \left\{|f(x)|:\|x\|_{\infty} \leq K_{3}\right\}, \\
K_{5}=\max \left\{|g(t, x)|+|e(t)|: t \in[0,2 \pi],\|x\|_{\infty} \leq K_{3}\right\} .
\end{gathered}
$$

From (3.16), there exists $t_{2} \in[0,2 \pi]$ such that $x^{\prime}\left(t_{2}\right)=0$, which implies that $\phi_{p}\left(x^{\prime}\left(t_{2}\right)\right)=0$. Therefore, integrating the both sides of $(3.3)$ over $\left[t_{2}, t\right]$, we have

$$
\phi_{p}\left(x^{\prime}(t)\right)=-\lambda \int_{t_{2}}^{t} f(x(t)) x^{\prime}(t) d t-\lambda \int_{t_{2}}^{t} g(t, x(t)) d t+\lambda \int_{t_{2}}^{t} e(t) d t
$$

Thus, we get from (3.8) that

$$
\begin{aligned}
\left|\phi_{p}\left(x^{\prime}(t)\right)\right| & \leq K_{4} \int_{0}^{2 \pi}\left|x^{\prime}(t)\right| d t+2 \pi K_{5} \\
& \leq K_{4}(2 \pi)^{1 / q}\left\|x^{\prime}\right\|_{p}+2 \pi K_{5} \\
& \leq(2 \pi)^{1 / q} K_{4} K_{1}+2 \pi K_{5}:=K_{6}^{p-1}, \quad \forall t \in[0,2 \pi] .
\end{aligned}
$$


Noting that $\left|\phi_{p}\left(x^{\prime}(t)\right)\right|=\left|x^{\prime}(t)\right|^{p-1}$, we obtain that

$$
\left\|x^{\prime}\right\|_{\infty} \leq K_{6}
$$

Combining (3.21) with (3.25), we have

$$
\|x\|_{C^{1}} \leq T_{1}
$$

where $T_{1}=\max \left\{K_{3}, K_{6}\right\}$. The proof is complete.

Now we give the proof of Theorem 3.1.

Proof of Theorem 3.1. Setting

$$
\Omega=\left\{x \in C^{1, \pi}:\|x\|_{C^{1}}<T_{1}+1\right\} .
$$

Obviously, the set $\Omega$ is an open-bounded set in $C^{1, \pi}$ and zero element $\theta \in \Omega$.

From the definition of operator $N$, it is easy to see that

$$
(N x)(t+\pi) \equiv-(N x)(t), \quad \forall x \in C^{1, \pi} .
$$

Hence, the operator $N$ sends $C^{1, \pi}$ into $C^{0, \pi}$. Let us define the operator $F_{\lambda}: \bar{\Omega} \rightarrow C^{1, \pi}$ by

$$
F_{\lambda} x=J \phi_{q} J \lambda N x=\phi_{q}(\lambda) L_{p}^{-1} N x, \quad \lambda \in[0,1] .
$$

Obviously, the operator $F_{\mathcal{\lambda}}$ is completely continuous in $\bar{\Omega}$ and the fixed points of operator $F_{1}$ are the antiperiodic solutions of (1.4).

With this in mind, let us define the completely continuous field $h_{\lambda}(x): \bar{\Omega} \times[0,1] \rightarrow$ $C^{1, \pi}$ by

$$
h_{\lambda}(x)=x-F_{\curlywedge} x
$$

By (3.20), we get that zero element $\theta \notin h_{\lambda}(\partial \Omega)$ for all $\lambda \in[0,1]$. So that, the following LeraySchauder degrees are well defined and

$$
\operatorname{deg}\left(\mathrm{id}-F_{1}, \Omega, \theta\right)=\operatorname{deg}\left(h_{1}, \Omega, \theta\right)=\operatorname{deg}\left(h_{0}, \Omega, \theta\right)=\operatorname{deg}(\mathrm{id}, \Omega, \theta)=1 \neq 0 .
$$

Consequently, the operator $F_{1}$ has at least one fixed point in $\Omega$ by using Lemma 2.2. Namely, (1.4) has at least one antiperiodic solution. The proof is complete.

\section{Antiperiodic Solutions with Symmetry for (1.5)}

In this section, we will prove the existence of even antiperiodic solutions or odd antiperiodic solutions for (1.5). 
Theorem 4.1. Assume that

$\left(H_{2}\right)$ the functions $g(t, x)$ and $e(t)$ are even in $t$, that is,

$$
g(-t, \cdot)=g(t, \cdot), \quad e(-t)=e(t), \quad \forall t \in \mathbb{R}
$$

and the assumption $\left(H_{1}\right)$ is true. Then (1.5) has at least one even antiperiodic solution $x(t)$, that is, $x(t)$ satisfies

$$
x(t+\pi)=-x(t), \quad x(-t)=x(t), \quad \forall t \in \mathbb{R} .
$$

Proof. We consider the homotopic equation of (1.5) as follows:

$$
\left(\phi_{p}\left(x^{\prime}\right)\right)^{\prime}=-\lambda g(t, x)+\lambda e(t), \quad \lambda \in[0,1] .
$$

Define the operator $N_{0}: C_{0}^{1, \pi} \rightarrow L^{1}([0,2 \pi], \mathbb{R})$ by

$$
\left(N_{0} x\right)(t)=-g(t, x(t))+e(t), \quad \forall t \in \mathbb{R} .
$$

Obviously, the operator $N_{0}$ is continuous.

Basing on the proof of Theorem 3.1, for the possible even antiperiodic solution $x(t)$ of (4.3), there exists a prior bounds in $C_{0}^{1, \pi}$, that is, $x(t)$ satisfies

$$
\|x\|_{C^{1}} \leq T_{2}
$$

where $T_{2}$ is a positive constant independent of $\lambda$. So that, our problem is reduced to construct one completely continuous operator $G_{\curlywedge}$ in $C_{0}^{1, \pi}$ which sends $C_{0}^{1, \pi}$ into $C_{0}^{1, \pi}$, such that the fixed points of operator $G_{1}$ in some open-bounded set are the even antiperiodic solutions of (1.5).

With this in mind, let us define the following set:

$$
\Omega_{0}=\left\{x \in C_{0}^{1, \pi}:\|x\|_{C^{1}}<T_{2}+1\right\}
$$

Obviously, the set $\Omega_{0}$ is an open-bounded set in $C_{0}^{1, \pi}$ and zero element $\theta \in \Omega_{0}$.

By hypothesis $\left(H_{2}\right)$, it is easy to see that

$$
\left(N_{0} x\right)(-t) \equiv\left(N_{0} x\right)(t), \quad \forall x \in C_{0}^{1, \pi}
$$

Hence, the operator $N_{0}$ sends $C_{0}^{1, \pi}$ into $C_{0}^{0, \pi}$. Let us define the completely continuous operator $G_{\curlywedge}: \overline{\Omega_{0}} \rightarrow C_{0}^{1, \pi}$ by

$$
G_{\lambda} x=J_{1} \phi_{q} J_{0} \lambda N_{0} x=\phi_{q}(\lambda)\left(\left.L_{p}\right|_{D\left(L_{p}\right) \cap C_{0}^{0, \pi}}\right)^{-1} N_{0} x, \quad \lambda \in[0,1] .
$$


From the similar arguments in the proof of Theorem 3.1, we can prove that there exists at least one fixed point of operator $G_{1}$ in $\Omega_{0}$. Thus, (1.5) has at least one even antiperiodic solution. The proof is complete.

Theorem 4.2. Assume that

$\left(H_{3}\right)$ the function $g(t, x)$ is odd in $t, x$ and $e(t)$ is odd in $t$, that is,

$$
g(-t,-x)=-g(t, x), \quad e(-t)=-e(t), \quad \forall t, x \in \mathbb{R}
$$

and the assumption $\left(H_{1}\right)$ is true. Then (1.5) has at least one odd antiperiodic solution $x(t)$, that is, $x(t)$ satisfies

$$
x(t+\pi)=-x(t), \quad x(-t)=-x(t), \quad \forall t \in \mathbb{R} .
$$

Proof. We consider the homotopic equation (4.3) of (1.5). Define the operator $N_{1}: C_{1}^{1, \pi} \rightarrow$ $L^{1}([0,2 \pi], \mathbb{R})$ by

$$
\left(N_{1} x\right)(t)=-g(t, x(t))+e(t), \quad \forall t \in \mathbb{R} .
$$

Obviously, the operator $N_{1}$ is continuous.

Based on the proof of Theorem 3.1, for the possible odd antiperiodic solutions of (4.3), there exists a prior bounds in $C_{1}^{1, \pi}$. Hence, our problem is reduced to construct one completely continuous operator $P_{\lambda}$ in $C_{1}^{1, \pi}$ which sends $C_{1}^{1, \pi}$ into $C_{1}^{1, \pi}$, such that the fixed points of operator $P_{1}$ in some open-bounded set are the odd antiperiodic solutions of (1.5).

With this in mind, let us define the set as follows:

$$
\Omega_{1}=\left\{x \in C_{1}^{1, \pi}:\|x\|_{C^{1}}<T_{2}+1\right\} .
$$

Obviously, the set $\Omega_{1}$ is an open-bounded set in $C_{1}^{1, \pi}$ and zero element $\theta \in \Omega_{1}$.

From the hypothesis $\left(H_{3}\right)$, it is easy to see that

$$
\left(N_{1} x\right)(-t) \equiv-\left(N_{1} x\right)(t), \quad \forall x \in C_{1}^{1, \pi}
$$

Thus, the operator $N_{1}$ sends $C_{1}^{1, \pi}$ into $C_{1}^{0, \pi}$. Let us define the completely continuous operator $P_{\curlywedge}: \overline{\Omega_{1}} \rightarrow C_{1}^{1, \pi}$ by

$$
P_{\lambda} x=J_{0} \phi_{q} J_{1} \lambda N_{1} x=\phi_{q}(\lambda)\left(\left.L_{p}\right|_{D\left(L_{p}\right) \cap C_{1}^{0, \pi}}\right)^{-1} N_{1} x, \quad \lambda \in[0,1]
$$

By a similar way as the proof of Theorem 3.1, we can prove that there exists at least one fixed point of operator $P_{1}$ in $\Omega_{1}$. So that, (1.5) has at least one odd antiperiodic solution. The proof is complete. 


\section{Examples}

In this section, we will give some examples to illustrate our main results.

Consider the following second-order differential equation with $p$-Laplacian operator:

$$
\left(\phi_{4}\left(x^{\prime}\right)\right)^{\prime}+x^{2} x^{\prime}+g(t, x)=e(t)
$$

Example 5.1. Let

$$
g(t, x)=\frac{1}{2} \sin ^{2} t \cdot x^{3}, \quad e(t)=\cos t
$$

For $p=4$, by direct calculation, we can get $\lambda_{1}=3 / 4$. Choosing $\alpha(t)=(1 / 2) \sin ^{2} t$, then (5.1) satisfies the condition of Theorem 3.1. So it has at least one antiperiodic solution.

Moreover, the conditions of Theorem 4.1 are also satisfied. Thus (5.1) has at least one even antiperiodic solution.

Example 5.2. Let

$$
g(t, x)=\frac{1}{2} \sin ^{2} t \cdot x^{3}, \quad e(t)=\sin t
$$

We choose $\alpha(t)=(1 / 2) \sin ^{2} t$. Obviously, (5.1) satisfies all the conditions of Theorem 4.2. Hence it has at least one odd antiperiodic solution.

\section{Acknowledgments}

The authors would like to thank the referees very much for their helpful comments and suggestions. This research was supported by the National Natural Science Foundation of China (10771212), the Fundamental Research Funds for the Central Universities, and the Science Foundation of China University of Mining and Technology (2008A037).

\section{References}

[1] C. Ahn and C. Rim, "Boundary flows in general coset theories," Journal of Physics, vol. 32, no. 13, pp. 2509-2525, 1999.

[2] H. Kleinert and A. Chervyakov, "Functional determinants from Wronski Green functions," Journal of Mathematical Physics, vol. 40, no. 11, pp. 6044-6051, 1999.

[3] S. Aizicovici, M. McKibben, and S. Reich, "Anti-periodic solutions to nonmonotone evolution equations with discontinuous nonlinearities," Nonlinear Analysis. Theory, Methods $\mathcal{E}$ Applications, vol. 43, no. 2, pp. 233-251, 2001.

[4] M. Nakao, "Existence of an anti-periodic solution for the quasilinear wave equation with viscosity," Journal of Mathematical Analysis and Applications, vol. 204, no. 3, pp. 754-764, 1996.

[5] P. Souplet, "Optimal uniqueness condition for the antiperiodic solutions of some nonlinear parabolic equations," Nonlinear Analysis. Theory, Methods \& Applications, vol. 32, no. 2, pp. 279-286, 1998.

[6] Z. Lu, Travelling Tube, Shanghai Science and Technology Press, Shanghai, China, 1962.

[7] Y. Q. Chen, "On Massera's theorem for anti-periodic solution," Advances in Mathematical Sciences and Applications, vol. 9, no. 1, pp. 125-128, 1999. 
[8] Y. Yin, "Monotone iterative technique and quasilinearization for some anti-periodic problems," Nonlinear World, vol. 3, no. 2, pp. 253-266, 1996.

[9] A. R. Aftabizadeh, N. H. Pavel, and Y. K. Huang, "Anti-periodic oscillations of some second-order differential equations and optimal control problems," Journal of Computational and Applied Mathematics, vol. 52, no. 1-3, pp. 3-21, 1994.

[10] T. Chen, W. Liu, J. Zhang, and M. Zhang, "The existence of anti-periodic solutions for Liénard equations," Journal of Mathematical Study, vol. 40, no. 2, pp. 187-195, 2007 (Chinese).

[11] B. Liu, "Anti-periodic solutions for forced Rayleigh-type equations," Nonlinear Analysis. Real World Applications, vol. 10, no. 5, pp. 2850-2856, 2009.

[12] W. B. Liu, J. J. Zhang, and T. Y. Chen, "Anti-symmetric periodic solutions for the third order differential systems," Applied Mathematics Letters. An International Journal of Rapid Publication, vol. 22, no. 5, pp. 668-673, 2009.

[13] Z. Luo, J. Shen, and J. J. Nieto, "Antiperiodic boundary value problem for first-order impulsive ordinary differential equations," Computers $\mathcal{E}$ Mathematics with Applications, vol. 49, no. 2-3, pp. 253261, 2005.

[14] H. L. Chen, "Antiperiodic wavelets," Journal of Computational Mathematics, vol. 14, no. 1, pp. 32-39, 1996.

[15] K. Wang and Y. Li, "A note on existence of (anti-)periodic and heteroclinic solutions for a class of second-order odes," Nonlinear Analysis. Theory, Methods E Applications, vol. 70, no. 4, pp. 1711-1724, 2009.

[16] L. S. Leibenson, "General problem of the movement of a compressible fluid in a porous medium," Izvestiia Akademii Nauk Kirgizskoŭ SSSR, vol. 9, pp. 7-10, 1983 (Russian).

[17] D. Jiang and W. Gao, "Upper and lower solution method and a singular boundary value problem for the one-dimensional p-Laplacian," Journal of Mathematical Analysis and Applications, vol. 252, no. 2, pp. 631-648, 2000.

[18] L. F. Lian and W. G. Ge, “The existence of solutions of m-point $p$-Laplacian boundary value problems at resonance," Acta Mathematicae Applicatae Sinica, vol. 28, no. 2, pp. 288-295, 2005.

[19] B. Liu and J. S. Yu, "On the existence of solution for the periodic boundary value problems with p-Laplacian operator," Journal of Systems Science and Mathematical Sciences, vol. 23, no. 1, pp. 76-85, 2003.

[20] J. Zhang, W. Liu, J. Ni, and T. Chen, "Multiple periodic solutions of $p$-Laplacian equation with oneside Nagumo condition," Journal of the Korean Mathematical Society, vol. 45, no. 6, pp. 1549-1559, 2008

[21] Y. Chen, J. J. Nieto, and D. O’Regan, "Anti-periodic solutions for fully nonlinear first-order differential equations," Mathematical and Computer Modelling, vol. 46, no. 9-10, pp. 1183-1190, 2007.

[22] G. Croce and B. Dacorogna, "On a generalized Wirtinger inequality," Discrete and Continuous Dynamical Systems, vol. 9, no. 5, pp. 1329-1341, 2003.

[23] K. Deimling, Nonlinear Functional Analysis, Springer, Berlin, Germany, 1985.

[24] R. E. Gaines and J. L. Mawhin, Coincidence Degree and Nonlinear Differential Equations, Lecture Notes in Mathematics, Vol. 568, Springer, Berlin, Germany, 1977. 